\title{
The COVID-19 Era and the Journal
}

The present issue of the Euroasian Journal of Hepato-Gastroenterology would be published after completing one decade of uninterrupted publication. Also, the present issue is going to be published during a pandemic of coronavirus disease 2019 (COVID-19). The countries of all the authors have mostly been under lockdown or passing through several restrictions those have severely compromised the opportunities of scientific studies. Also, the publisher has been working in an unusual state with nation-wise lockdown and several allied problems. Even then, the timely publication of the present issue of the journal represents tremendous efforts and planned activities of the authors, publishers, reviewers and editors. The publication became possible due to a concerted programming in this period of international emergency.

As for the journal itself, it is progressing and has adapted an article submission system that is completely based on electronic submission procedures. The reviewers and the editors have accomplished their duties via journal DASH BOARD with their whole-hearted support.

The region of Eurasia consists of bunch of countries those are generally highly populated, with a developing economy and many of these countries are resource-constrained. The pandemic of COVID-19 has affected all countries of Euroasian region in diverse ways with severe involvement in some countries and the worst are yet to appear in others. However, getting rid of COVID-19 pandemic is yet to be visible.

Although this journal is primarily devoted to the disciplines of Gastroenterology and Hepatology, the pandemic of COVID-19 has expanded its nature (may be for temporarily), and articles dealing with COVID-19 have been enrolled in this issue of the journal. The role of COVID-19 on gastroenterological and hepatological diseases may be immense. On the other hand, gastroenterological and hepatological diseases may also influence the pathogenesis of COVID-19. These are pending issues for our disciplines and possibly all disciplines of medical sciences. We have to live with COVID-19 for months and possibly years.

The journal will modify its nature and policy based on the reality of the containment and complications of COVID-19 with an eye on the discipline of Gastroenterology and Hepatology.

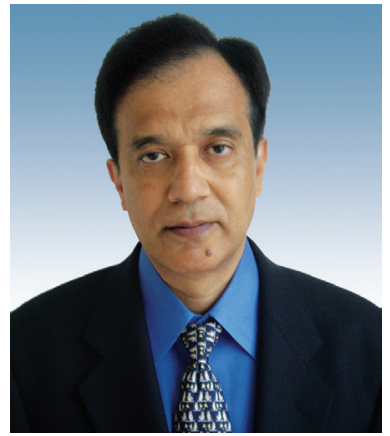

Sheikh Mohammad Fazle Akbar

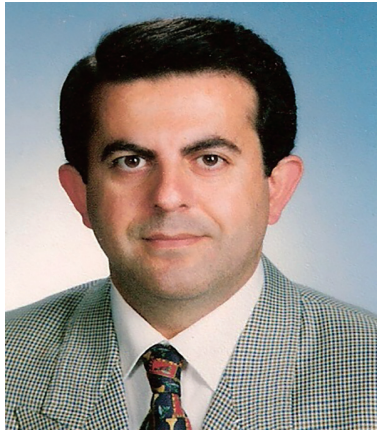

Hasan Ozkan

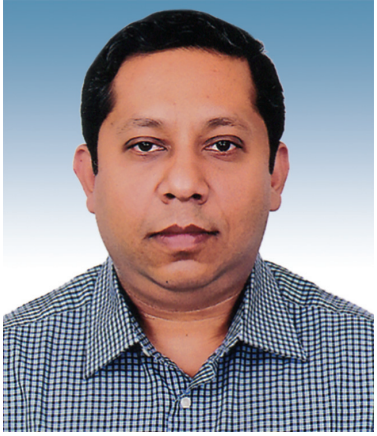

Mamun Al Mahtab 